\title{
Endoplasmic Reticulum Stress in the Kidney as a Novel Mediator of Kidney Injury
}

\author{
Reiko Inagi \\ Division of Nephrology and Endocrinology, University of Tokyo School of Medicine, Tokyo, Japan
}

\section{Key Words \\ Chronic kidney disease $\cdot$ Acute kidney injury $\cdot$ GRP78 • \\ IRE1 • PERK · ATF6 $\cdot$ Oxidative stress $\cdot$ Inflammation}

\begin{abstract}
The endoplasmic reticulum (ER) plays an important role in the maintenance of protein homeostasis through its control of the concentration, conformation, folding, and trafficking of client proteins. Disturbances such as hypoxia, glucose depletion, and oxidative stress may lead to ER dysfunction, which can induce ER stress and the subsequent unfolded protein response (UPR). The UPR initially serves as an adaptive response, but will also induce apoptosis in cells under severe or prolonged ER stress. Oxidative stress and inflammation are compounded by ER stress via the UPR, suggesting the potential pathophysiological significance of this response across a wide range of diseases. Accumulating evidence indicates that ER stress contributes to glomerular and tubular damage in patients with acute and chronic kidney disease. In glomeruli, podocyte or mesangial dysfunction tends to induce the adaptive UPR, which involves ER chaperone expression and the attenuation of protein translation, to maintain ER homeostasis and ensure cell survival. In tubules, apoptosis resulting from epithelial cell damage is caused, at least in part, by the proapoptotic UPR. These findings emphasize the possibility of the development of novel renoprotective drugs which target ER stress.
\end{abstract}

Copyright $\odot 2009$ S. Karger AG, Basel

\section{Endoplasmic Reticulum, Endoplasmic Reticulum Stress, and the Unfolded Protein Response}

The endoplasmic reticulum (ER) performs several cellular functions including the regulation of protein biosynthesis, folding, trafficking, and modification. ER functions are sensitive to environmental insults such as ischemia, glucose deprivation, oxidative stress, or genetic mutation which can cause aberrant protein folding. The accumulation of these aberrant unfolded and malfolded proteins in the ER lumen in turn induces a range of ER dysfunctions, collectively referred to as ER stress [1-3]. To ensure the fidelity of protein folding and prevent the accumulation of unfolded or malfolded proteins, cells experiencing ER stress invoke a well-conserved adaptive response, known as the unfolded protein response (UPR), which is initially aimed at ameliorating the damage but can eventually trigger cell death if the ER stress is severe or prolonged (fig. 1).

The primary purpose of the UPR is to facilitate adaptation to the changing environment which evokes it and reestablish normal ER function. Some UPR pathways enhance the protein-folding capacity by activating the transcription of UPR target genes such as $\mathrm{Ca}^{2+}$-dependent molecular chaperones, including glucose-regulated protein-78 (GRP78), GRP94, and calreticulin [4]. GRP78, also referred to as $\mathrm{BiP}$, is a central regulator of ER function. The N-termini of transmembrane ER proteins such as inositol-requiring enzyme-1 (IRE1), double-stranded

\section{KARGER}

Fax +41613061234 E-Mail karger@karger.ch www.karger.com (c) 2009 S. Karger AG, Basel

$1660-2129 / 09 / 1121-0001 \$ 26.00 / 0$

Accessible online at:

www.karger.com/nee
Reiko Inagi

Division of Nephrology and Endocrinology

University of Tokyo School of Medicine

7-3-1, Hongo Bunkyo-ku, Tokyo 113-8655 (Japan)

Tel. +81 33815 5411, Fax +81 35800 8806, E-Mail inagi-npr@umin.ac.jp 

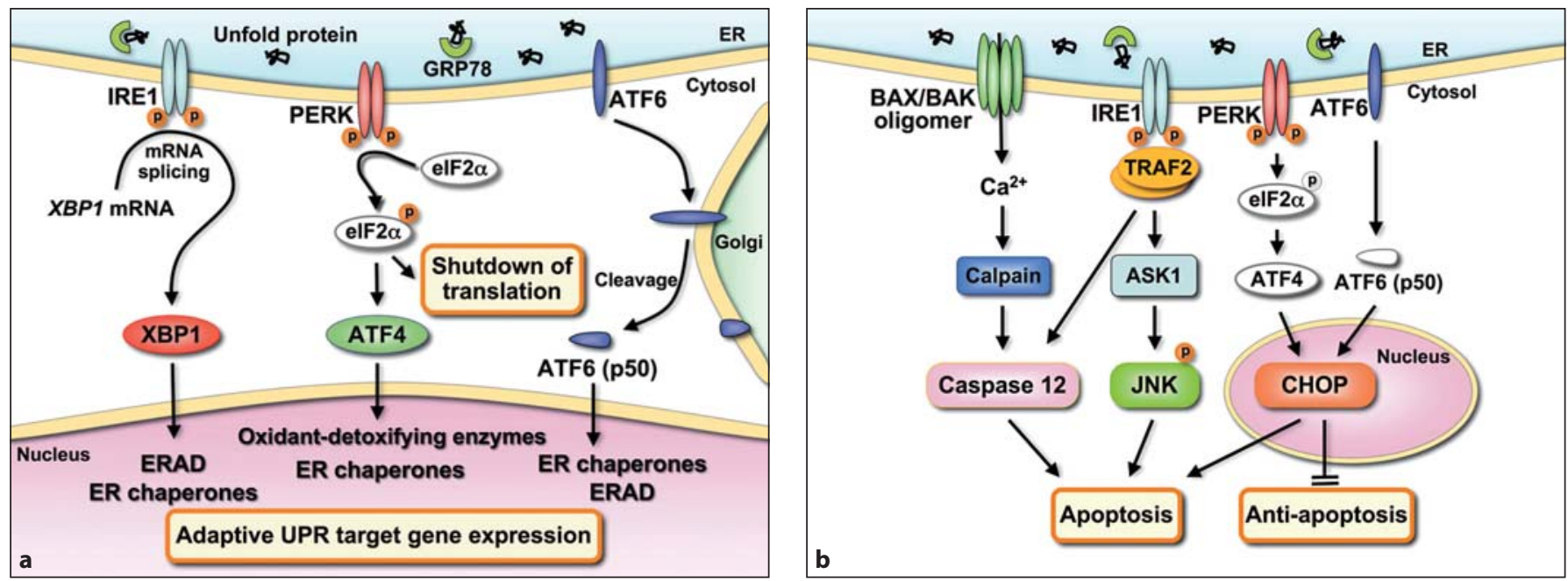

Fig. 1. To keep cells from dying under ER stress conditions, the IRE1, PERK, or ATF6 pathway induces the adaptive UPR, which includes promotion of the expression of genes encoding ER chaperones, ERAD components, or oxidant-detoxifying enzymes (a). Cells rendered dysfunctional due to severe or prolonged ER stress are eliminated from the organism by proapoptotic UPR mediated by CHOP, JNK, or caspase 12 (b).

RNA-activated protein kinase-like ER kinase (PERK), and activating transcription factor-6 (ATF6) are normally held in an inactive state in the ER membranes by binding to GRP78. When unfolded proteins accumulate in the ER lumen, GRP78 releases the transmembrane ER proteins, allowing them to either dimerize or move to other locations within the cell, thereby initiating UPR signaling to reestablish homeostasis and normal ER function. This process occurs through the following pathways.

\section{IRE1 Pathway}

The IRE1 pathway mainly induces expression of UPR target genes in charge of ER-associated degradation (ERAD), which serve as an adaptive response to ER stress by degrading misfolded proteins [5]. IRE1 is activated by dimerization after dissociation from GRP78, and its RNAase activity initiates mRNA splicing in X-box-binding protein-1 (XBP1), thereby creating a potent transcriptional activator of genes which encode ERAD components. The IRE1-XBP1 pathway also induces the transcription of genes encoding ER chaperones such as GRP78, GRP94, and calreticulin [6].

\section{PERK Pathway}

The PERK pathway alleviates ER burden by reducing the frequency of initiation of mRNA translation and thus decreasing the influx of new proteins into the ER. PERK is a Ser/Thr protein kinase, and its active homodimer phosphorylates and inactivates eukaryotic initiation factor- $2 \alpha$ (eIF $2 \alpha$ ), thereby shutting off protein translation globally and reducing protein load on the ER [2]. The PERK pathway not only attenuates translation, but also selectively activates the transcription of UPR-inducible genes, including ER chaperones, and antioxidant and oxidant-detoxifying enzymes, such as glutathione S-transferase and hemoxygenase-1 (HO-1), to protect cells from both oxidative as well as ER stress [7].

\section{ATF6 Pathway}

ATF6, similar to active XBP1, is a regulatory protein which promotes UPR-inducible gene expression. After dissociating from GRP78, ATF6 is transported to the Golgi and cleaved by site- 1 and site- 2 proteases (S1P and S2P). The cleaved cytosolic fragment of ATP6 translocates to the nucleus, where it subsequently activates the transcription of its target genes, which encode ER chaperones and ERAD components $[6,8]$.

\section{ER Stress-Induced Apoptosis when the Adaptive UPR Is Overwhelmed}

When cells under excessive or prolonged ER stress fail to resolve the protein-folding defect and restore homeostasis in the ER via the adaptive UPR pathway, apoptosis is triggered. This dispensation with dysfunctional cells 
Table 1. Pathogenic contribution of ER stress in kidney disease

\begin{tabular}{lll}
\hline Sites & ER stress states & References \\
\hline Podocyte & Passive Heymann nephritis & 17,18 \\
& Puromycin nephrosis & 19 \\
& Mislocalization of slit diaphragm & 20 \\
& components (mutation of nephrin) & \\
& Megsin overexpression & 15 \\
& Advanced glycated BSA & 62 \\
\hline Mesangium & Thy-1 nephritis & 21 \\
\hline Tubular & Puromycin nephropathy & 24 \\
epithelium & Chronic BSA-overload nephropathy & 28 \\
& Ischemia-reperfusion & $25-27,54$ \\
& Established diabetic nephropathy & 29 \\
& Minimal-change disease & 29 \\
& Nephrotoxic drugs (antibiotics, immu- & $10,21,23$, \\
& nosuppressants, and anticancer drugs) & $30-33$ \\
\hline
\end{tabular}

represents a last resort on the part of multicellular organisms $[2,9]$. ER stress-induced apoptosis is mediated by several pathways as follows (fig. 1).

\section{CCAAT/Enhancer-Binding Protein Homologous \\ Protein-Mediated Pathway}

ER stress-induced apoptosis is mainly mediated by CCAAT/enhancer-binding protein homologous protein (CHOP), also referred to as growth arrest and DNA damage 153 (GADD153). CHOP is a transcription factor, which induces several proapoptotic factors, and is downstream of the PERK (PERK-eIF2 $\alpha$ signal followed by ATF4 expression) and ATF6 UPR pathways. CHOP also downregulates anti-apoptotic $\mathrm{Bcl}-2$, leading to enhanced oxidant injury and apoptosis. These findings are consistent with a previous observation that overexpression of Bcl-2 specifically in the ER protected renal tubular cells against ER stress-induced apoptosis [10]. The activity of $\mathrm{CHOP}$ in inducing apoptosis is dependent on the duration and degree of ER stress. Severe ER stress preferentially induces proapoptotic $\mathrm{CHOP}$ expression as compared to mild ER stress.

\section{IRE1-Mediated Pathway}

The cytoplasmic domain of activated (dimerized) IRE1 interacts with the adaptor factor, tumor necrosis factor receptor-associated factor-2 (TRAF2), leading to apoptosis. The IRE1-TRAF2 complex activates the c-Jun N-terminal kinase (JNK) pathway via apoptosis signalregulating kinase-1 (ASK1) phosphorylation. IRE1-
TRAF2 also activates the caspase-12-dependent apoptotic pathway as well as the JNK-mediated apoptotic pathway.

\section{Caspase-Mediated Pathway}

Several components of the caspase cascade are reported to be involved in ER stress-induced apoptosis. In particular, caspase-12, which is associated with the ER membrane, is a proximal regulator for ER stress-induced caspase activation followed by apoptosis. Activated caspase-12 cleaves procaspase- 9 to activate procaspase-3. In addition to the IRE1-TRAF2 pathway described above, the BAX-BAK pathway also activates caspase-12-mediated apoptosis. Under ER stress conditions, proapoptotic $\mathrm{BAX}$ and $\mathrm{BAK}$ at the ER membrane cause the release of $\mathrm{Ca}^{2+}$ from the ER; the resulting increase in cytosolic $\mathrm{Ca}^{2+}$ activates calpain, which cleaves procaspase- 12 to its active form. Because caspase-12 is expressed in rodents but not primates, caspase- 4 is instead thought to contribute to ER stress-induced apoptosis in human cells.

\section{Kidney Diseases Associated with ER Stress}

ER stress plays a pathogenic role in diseases associated with the accumulation of misfolded proteins, such as conformational diseases like Alzheimer's, Parkinson's, and Huntington's diseases. Further, ER stress is also associated with a wide range of other conditions, including ischemia/reperfusion injury, diabetes, and atherosclerosis $[3,11-14]$. Accumulating evidence, including our previous studies, suggests a pathophysiological role of ER stress in the kidney (table 1).

To investigate the role of megsin, a novel molecule identified by our laboratory, we established transgenic rats overexpressing megsin. The rats developed severe proteinuria at an early age, and detailed pathological analysis revealed that excessive accumulation of transgene products in the ER of podocytes induced ER stress and subsequent kidney failure. Further, the rats showed pathological features similar to those observed in human patients with serpinopathy, a conformational disease caused by misfolded or aggregated serine protease inhibitor (serpin) in the ER $[15,16]$. Megsin is a novel serpin, and thus excessive production can overwhelm the protein-folding capacity of the ER and induce accumulation of misfolded megsin, in turn leading to serpinopathy associated with ER stress. These observations led us to investigate the pathological role of ER stress in several kidney diseases. 


\section{Glomerular Injury}

Podocytes play a crucial role in the development of glomerulosclerosis. Previous reports have identified the induction of ER stress in podocytes in models of membranous nephropathy (Heymann nephritis) $[17,18]$ and focal segmental or minimal-change nephrotic syndrome (puromycin nephrosis) [19]. Among examples, complementmediated podocyte injury induced an adaptive UPR pathway such as the expression of ER chaperones (GRP78 and GRP94) in proteinuric rats with Heymann nephritis. In puromycin nephrosis rats, nephrotic-range proteinuria increased GRP78 expression in podocytes and led to the mislocalization of nephrin to the cytoplasm, demonstrating that proteinuria-induced podocyte damage was associated with ER stress, which in turn affected the biogenesis of slit diaphragm proteins. Further, in vitro studies utilizing human embryonic kidney-293 cells expressing full-length human nephrin have emphasized the pathogenic contribution of ER stress in podocytes. Energy depletion evokes a rapid increase in ER stress, leading to the formation of underglycosylated nephrin, in turn suggesting that ER stress in podocytes may cause the alteration of nephrin $\mathrm{N}$-glycosylation as an underlying factor in the pathomechanism of proteinuria [20].

In our previous study of mesangial injury in a rat model of mesangioproliferative glomerulonephritis, we demonstrated that the UPR pathway is induced in the glomeruli of these rats, including the induction of ER chaperones (GRP78 and 150-kDa oxygen-regulated protein, ORP150) and the attenuation of protein translation via the PERK-eIF $2 \alpha$ pathway [21].

\section{Tubulointerstitial Injury}

A final common pathway to end-stage kidney disease is expected to be found in the tubulointerstitium. Tubular cells are particularly sensitive to ER stress inducers such as tunicamycin, which disturbs protein glycosylation in the ER and causes ER stress. As evidence of this, mice injected with tunicamycin develop acute renal tubular necrosis [22]; while in parallel, mice lacking the anti-apoptotic protein Bax inhibitor-1 (BI-1) in the ER are more sensitive to tunicamycin-induced renal tubule cell death [23]. Given these findings, we investigated the effect of massive proteinuria, which mediates tubulointerstitial injury in various chronic kidney diseases, by utilizing puromycin nephropathy in rats [24]. The findings demonstrate that protein load in tubular cells invoked ER stressinduced apoptosis via the caspase-12 pathway.

Chronic hypoxia, another important mediator of tubulointerstitial injury, leads to end-stage kidney disease.
While the effects of chronic hypoxia on tubular ER stress have not been elucidated, other investigations have successfully shown the induction of ER stress in tubular cells subjected to acute ischemia [25-27]. The major role played by the ER stress response in chronic tubulointerstitial injury was emphasized in a recent study examining knockin mice expressing mutant GRP78: heterozygous mutant GRP78 mice showed significant tubulointerstitial lesions with aging [28]. Further, proteinuria induced by chronic protein overload accelerated lesion development, together with caspase- 12 activation and tubular cell apoptosis. These results confirmed that the ER stress pathway is significantly involved in the pathophysiology of chronic kidney injury with tubulointerstitial damage.

The body of evidence obtained from in vivo and in vitro animal experiments, including our results, is consistent with clinical observations in humans. The adaptive URP pathways and ER stress-induced apoptosis are invoked in the tubular epithelium of patients suffering from established diabetic nephropathy or minimal change disease, suggesting that hyperglycemia and proteinuria can induce ER stress in tubules in both human and rodents [29].

Nephrotoxic drugs, such as cisplatin or gentamicin, are known to induce ER stress in the kidney [30-32]. Further, recent studies have shown that ER stress in turn induces autophagy in tubules in cyclosporine nephropathy [33]. Autophagy is a catabolic process involving the degradation of a cell's own components through the lysosomal machinery, and is a regulated process that plays a normal part in cell growth, development, and homeostasis by helping to maintain a balance between the synthesis, degradation, and subsequent recycling of macromolecules and organelles. Autophagy is also known to commit the cell to a third type of cell death besides apoptosis and necrosis. Further studies are needed to elucidate the role of autophagy in kidney diseases associated with ER stress.

\section{Pathological States Associated with ER Stress: A Link to Oxidative Stress and Inflammation}

Although protein-folding defects in the ER due to mutations associated with protein misfolding or increased client protein synthesis are known to contribute to ER stress in conformational diseases, several other factors also trigger protein-folding defects leading to ER stress in other diseases. Hypoxia and ischemia, for example, are known ER stressors: the hypoxic microenvironment 
brought on poor vascularization in tumors bringing about physiological ER stress. The UPR is crucial to the survival of tumor cells [34]. GRP78, for example, is induced in a wide range of human cancers, and its expression level correlates with tumor progression, metastasis, and drug resistance [35].

While the UPR promotes the survival of malignant cells, recent evidence strongly suggests the paradoxical involvement of ER stress as an initiator of cellular death signaling during hypoxia and ischemia/reperfusion insults in various organs $[36,37]$. Ischemia or ischemia/reperfusion of the brain or heart induced several UPR pathways, which subsequently lead to adaptive and organprotective UPR (brief periods of stress) or apoptosis (under prolonged severe stress) [38-40]. Further, analysis of coronary artery segments from affected patients showed a marked increase in ER chaperone expression and apoptotic cells in smooth muscle cells as well as macrophages in the fibrous caps of thin-cap atheroma and ruptured plaques, but not in the those of thick-cap atheroma and fibrous plaques, suggesting that ER stress plays a pathogenic role in the rupture of atherosclerotic plaques [41].

Several factors contribute to ER stress under hypoxic conditions. Hypoxia is known to initiate protein misfolding in the ER, thereby diverting resident ER chaperones and freeing transmembrane signal-transducing proteins of the ER to initiate the UPR. In addition, reperfusion triggers oxidative stress with production of reactive oxygen species (ROS), altering cellular redox-dependent reactions and interfering with protein disulfide bonding, ultimately resulting in protein misfolding in the ER. Studies utilizing overexpression of antioxidative stress enzymes have emphasized the role of oxidative stress in the induction of ER stress. The ischemia-induced ER stress response was markedly less pronounced in animals overexpressing copper/zinc superoxide dismutase $(\mathrm{Cu} /$ $\mathrm{Zn}$-SOD), suggesting that superoxide radicals play a role in this pathological process [42]. Further, cadmium caused generation of ROS with subsequent induction of ER stress, leading to apoptosis in a cultured renal proximal tubular cell line; this cadmium-induced ER stress and apoptosis were significantly attenuated by transfection with manganese SOD [43]. Of particular note, recent studies have demonstrated that the accumulation of intracellular ROS caused by ER stress is attenuated by the PERK pathway of the UPR, which activates the transcription factor, nuclear factor erythroid-derived-2-related factor-2 (Nrf2), and maintains redox homeostasis, thereby ensuring cell survival. The antioxidant effects of the
PERK pathway are supported by the finding that PERKdeficient cells exposed to tunicamycin, an ER stress inducer, showed a toxic accumulation of intracellular ROS compared to wild-type cells [44].

Nitric oxide (NO), produced in excessive levels following ischemia, also contributes to ER stress. Ischemia-induced activation of PERK and phosphorylation of eIF $2 \alpha$ are completely blocked in animals with targeted deletion of the endothelial or neuronal NO synthase (NOS) gene [45]. The notion that NO plays a role in ischemia-induced ER stress is supported by the observation that $\mathrm{ER} \mathrm{Ca}^{2+}$ stores were depleted after an ischemic episode. This depletion occurs due to $\mathrm{Ca}^{2+}$ leakage into the cytosol and subsequent uptake into mitochondria, resulting in mitochondrial ROS generation. Recovery of ER $\mathrm{Ca}^{2+}$ homeostasis was observed only in animals pretreated with neuroprotective levels of an NOS inhibitor [46]. The excessive NO production seen in hypoxia, which alters calcium homeostasis in both the ER and mitochondria, may initiate a vicious cycle of ER stress, oxidative stress, and apoptosis.

Accumulating evidence indicates the involvement of ER stress in several types of inflammation [47]. In inflammation of the central nervous system, for example, interferon- $\gamma$ induces ER stress and apoptosis of oligodendrocytes [48], while in lipopolysaccharide-induced inflammation of the lungs, lipopolysaccharide induces ER stress and $\mathrm{CHOP}$ expression, leading to the apoptosis of lung cells [37]. At the same time, nuclear factor- $\kappa \mathrm{B}(\mathrm{NF}-\kappa \mathrm{B})$, a key transcriptional regulator of genes involved in the inflammatory response, is activated by ER stress, an action caused by translational attenuation and degradation of

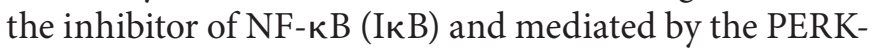
eIF2 $\alpha$ and IRE1-TRAF2 pathways, respectively [47].

ER stress is also involved in autoimmunity. A microarray analysis of muscle tissue obtained from patients with myositis revealed the induction of GRP78, CHOP, and GADD45 expression in the cells, suggesting that the ER stress response is responsible for the skeletal muscle damage and dysfunction evident in autoimmune myositis [49]. Intestinal inflammation has been shown to result from XBP1 abnormalities in intestinal epithelial cells, suggesting a link between cell-specific ER stress and organ-specific inflammation [50]. In intestinal epithelial cells, $\mathrm{Xbp} 1$ deletion results in spontaneous enteritis, while mutant variants of the gene are associated with both forms of human inflammatory bowel disease (Crohn's disease and ulcerative colitis).

The UPR pathway links not only Nrf2 and NF-кB pathways but also the mammalian target of rapamycin 
Table 2. Therapeutic modality targeting ER stress

\begin{tabular}{|c|c|c|}
\hline Modality & Function(s) & References \\
\hline \multicolumn{3}{|c|}{ ER chaperone overexpression } \\
\hline GRP78 & Translation shutdown $\uparrow$ & 52 \\
\hline ORP150 & Protein folding $\uparrow$ & 26 \\
\hline \multicolumn{3}{|c|}{ ER stress preconditioning } \\
\hline ER stress inducers & Basal adaptive UPR $\uparrow$ & $21,53,54$ \\
\hline \multicolumn{3}{|l|}{ Chemical compounds } \\
\hline DTTox & GRP78 expression $\uparrow$ & 55 \\
\hline BIX & GRP78 expression $\uparrow$ & 38 \\
\hline Salubrinal & Adaptive UPR (PERK/eIF2 $\alpha) \uparrow$ & 56 \\
\hline Benzodiazepinones & IRE1/TRAF2/ASK1 $\downarrow$ & 57 \\
\hline \multicolumn{3}{|c|}{ Chemical chaperones } \\
\hline $4-\mathrm{PBA}$ & $\begin{array}{l}\text { Protein folding } \uparrow, \text { ERAD } \uparrow \text {, } \\
\text { caspase } 12 \downarrow\end{array}$ & 58,59 \\
\hline TUDCA & $\begin{array}{l}\text { Adaptive UPR } \uparrow \text {, proapoptotic } \\
\text { UPR } \downarrow\end{array}$ & $60-62$ \\
\hline \multicolumn{3}{|l|}{ Others } \\
\hline TM2002 & Oxidative stress $\downarrow$ & 63 \\
\hline Mizoribine & $\begin{array}{l}\text { Intracellular energy for protein } \\
\text { folding } \uparrow\end{array}$ & 19 \\
\hline
\end{tabular}

(mTOR) pathway, which regulates protein synthesis and cell growth and survival. mTOR activation causes ER stress by an increase in client protein load in the ER, subsequently the activated UPR (JNK pathway) downregulates mTOR activation [51]. The importance of this negative feedback loop between the mTOR and the UPR pathways remains elusive. However, $\mathrm{mTOR}$ and UPR are $\mathrm{O}_{2}$-sensitive signaling pathways as is the HIF pathway. Thus, hypoxic cell phenotype may be composed of the responses orchestrated by these pathways.

\section{Treatment Targeting ER Stress}

Several studies support the idea that artificial modulation of ER stress may provide protection to the cell. Cells overexpressing GRP78 are resistant to conditions associated with ER stress [52]. Further, systemic overexpression of ORP150 protects the kidney against ischemia/reperfusion injury [26]. However, while gene transfer may be promising for future therapy, other therapeutic modalities are needed to address immediate concerns (table 2).

Preconditioning was first identified by the observation that in ischemic diseases brief ischemic treatment before the subsequent insult induced a state of resistance to blood loss by initiating a cascade of biochemical events, allow- ing for the upregulation of the cellular protective genes in the tissues. In our previous study, we observed beneficial effects of preconditioning with ER stress by utilizing a subnephritogenic dose of the ER stress inducer (tunicamycin or thapsigargin) in a model of mesangioproliferative glomerulonephritis [21]. Given that overwhelming ER stress leads to eventual cell death via proapoptotic UPR including activation of caspase-12, induction of ER stress as a therapeutic approach may seem paradoxical. However, ER stress also induces the adaptive UPR, shutting down translation and expressing ER chaperones to aid in protein folding, which improves the efficiency of energy consumption. Cytoprotection by preconditioning targeting ER stress has also been confirmed in cultured renal tubular cells treated with clinically relevant nephrotoxic drugs and in tubular injury in mice with renal ischemia-reperfusion [53, 54]. Thus, preconditioning targeting ER stress enhances the adaptive UPR, thereby protecting the cell more effectively against the pathogenic environment via maintenance of ER function.

Chemical compounds targeting the ER stress pathways have been shown to be effective across a range of situations, building enthusiasm for the therapeutic potential latent in ER stress response augmentation. One study found that trans-4,5-dihydroxy-1,2-dithiane (DTTox) stimulated an increase in GRP78 expression and protected the proximal tubular epithelium against a nephrotoxic chemical [55]. A GRP78 reporter assay system identified the chemical compound $\mathrm{BiP}$ inducer $\mathrm{X}$ (BIX, 1-(3,4-dihydroxyphenyl)-2-thiocyanato-ethanone) as an inducer of GRP78 mRNA. In gerbils subjected to forebrain ischemia, prior treatment with BIX protected against disease manifestation [39]. Further, screening for inhibitors of ER stress-induced neuronal death identified the chemical compound salubrinal, which suppresses protein phosphatases responsible for the dephosphorylation of eIF $2 \alpha$, thereby increasing the accumulation of phosphorylated eIF $2 \alpha$ and providing protection from the apoptosis induced by several ER stress inducers [56]. Kim et al. [57] used a cell-based high throughput screening assay to identify components that halted ER stress-induced apoptosis. Results showed that benzodiazepinones selectively enhanced ASK1 phosphorylation at serine-967, which reduces but does not induce ASK1 activity, resulting in inhibition of the IRE1-ASK1 pathway. These findings suggest the usefulness of a modulator of ASK1 function as an ER stress-induced apoptosis inhibitor [57].

Alternatively, chemical chaperones present another possible treatment method. Chemical chaperones such as sodium 4-phenylbutyrate (4-PBA) are a group of com- 
pounds known to improve ER folding capacity and facilitate the trafficking of unfolded or malfolded proteins by stabilizing their conformation. In one study, pre- or post-treatment with 4-PBA at therapeutic doses attenuated infarction volume, hemispheric swelling, and apoptosis, and also improved neurological status in a mouse model of brain ischemia, possibly due to a decrease in the protein load retained by the ER [58]. Mutated nephrin associated with misfolding and mislocalization is rescued by 4-PBA, suggesting the beneficial effect of ER stress modulators in nephrosis induced by nephrin mutation [59]. Endogenous bile acid derivatives, such as tauroursodeoxycholic acid (TUDCA), can also modulate ER function, protecting liver cells against ER stress-induced apoptosis or restoring glucose homeostasis in type 2 diabetic mice $[60,61]$. Unlike 4-PBA, however, TUDCA has not been shown to act as a chaperone that promotes the folding and trafficking of unfolded or malfolded proteins. Instead, TUDCA is likely to increase the stability of unfolded or malfolded proteins, possibly as a result of its influence on UPR activation with subsequent enhancement of the ERAD pathway. ER stress plays a role in advanced glycation end product-induced apoptosis in murine podocytes, and TUDCA prevents apoptosis by blocking an ER stress-mediated apoptotic pathway [62].

In addition to these compounds which enhance adaptive UPR or suppress proapoptotic UPR, effective attenuation of the ER stress response has also been observed in compounds which suppress oxidative stress. TM2002, an inhibitor of oxidative protein glycation, shows renoprotective effects associated with a reduction in ER stress in ischemic-reperfusion rats [63]. Mizoribine, an immuno- suppressant, is an inhibitor of purine nucleotide biosynthesis, subsequently restoring the intracellular energy balance during ER stress by salvaging ATP levels. Mizoribine treatment reduced ER stress and rescued the mislocalization of nephrin to the cytoplasm in podocytes with glucose starvation, suggesting that the remission of proteinuria by mizoribine is mediated by the reduction of ER stress [19].

\section{Conclusion}

ER stress stimulates adaptive UPR to restore ER homeostasis and the proapoptotic UPR to eliminate cells under prolonged stress. The balance of these two contrasting UPRs appears to depend on either or both ER stress status (severity or duration) or cell type. In acute and chronic kidney disease, ER stress contributes to the development and progression of glomerular and tubular disease, and accumulating evidence suggests the possibility of a novel therapeutic approach targeting ER stress. The finding that ER stress links oxidative stress and inflammation further highlights the potential benefit of maintaining ER homeostasis by modulating ER stress status to protect the kidney against various pathogenic environments.

\section{Acknowledgement}

This work was supported by Grants-in-Aid for Scientific Research from the Japan Society for the Promotion of Science (19590939) to R.I.

\section{References}

1 Kaufman RJ: Stress signaling from the lumen of the endoplasmic reticulum: coordination of gene transcriptional and translational controls. Genes Dev 1999;13: 1211-1233.

$\checkmark 2$ Malhotra JD, Kaufman RJ: Endoplasmic reticulum stress and oxidative stress: a vicious cycle or a double-edged sword? Antioxid Redox Signal 2007;9:2277-2293.

3 Yoshida H: ER stress and diseases. FEBS J 2007;274:630-658.

$\checkmark 4$ Ni M, Lee AS: ER chaperones in mammalian development and human diseases. FEBS Lett 2007;581:3641-3651.

5 Vembar SS, Brodsky JL: One step at a time: endoplasmic reticulum-associated degradation. Nat Rev Mol Cell Biol 2008;9:944-957.

Endoplasmic Reticulum Stress in the Kidney
6 Yamamoto K, Sato T, Matsui T, Sato M, Okada T, Yoshida H, Harada A, Mori K: Transcriptional induction of mammalian ER quality control proteins is mediated by single or combined action of ATF $6 \alpha$ and XBP1. Dev Cell 2007;13:365-376.

7 Zhang DD: Mechanistic studies of the Nrf2Keap1 signaling pathway. Drug Metab Rev 2006;38:769-789.

$\checkmark 8$ Adachi Y, Yamamoto K, Okada T, Yoshida H, Harada A, Mori K: ATF6 is a transcription factor specializing in the regulation of quality control proteins in the endoplasmic reticulum. Cell Struct Funct 2008;33:75-89.

$\checkmark 9$ Ron D, Walter P: Signal integration in the endoplasmic reticulum unfolded protein response. Nat Rev Mol Cell Biol 2007;8:519529.
10 Bhatt K, Feng L, Pabla N, Liu K, Smith S, Dong Z: Effects of targeted $\mathrm{Bcl}-2$ expression in mitochondria or endoplasmic reticulum on renal tubular cell apoptosis. Am J Physiol Renal Physiol 2008;294:F499-F507.

$\checkmark 11$ Zhang K, Kaufman RJ: The unfolded protein response: A stress signaling pathway critical for health and disease. Neurology 2006;66: S102-S109.

12 Oyadomari S, Mori M: Roles of CHOP/ GADD153 in endoplasmic reticulum stress. Cell Death Differ 2004;11:381-389.

$\checkmark 13$ Xu C, Bailly-Maitre B, Reed JC: Endoplasmic reticulum stress: cell life and death decisions. J Clin Invest 2005;115:2656-2664.

14 Ogawa S, Kitao Y, Hori O: Ischemia-induced neuronal cell death and stress response. Antioxid Redox Signal 2007;9:573-587. 
-15 Inagi R, Nangaku M, Onogi H, Ueyama H, 25 Kuznetsov G, Bush KT, Zhang PL, Nigam Kitao Y, Nakazato K, Ogawa S, Kurokawa K, Couser WG, Miyata T: Involvement of endoplasmic reticulum (ER) stress in podocyte injury induced by excessive protein accumulation. Kidney Int 2005;68:2639-2650.

16 Inagi R, Nangaku M, Usuda N, Shimizu A, Onogi H, Izuhara Y, Nakazato K, Ueda Y, Oishi H, Takahashi S, Yamamoto M, Suzuki D, Kurokawa K, van Ypersele de Strihou C, Miyata T: Novel serpinopathy in rat kidney and pancreas induced by overexpression of megsin. J Am Soc Nephrol 2005;16:1339-1349.

-17 Cybulsky AV, Takano T, Papillon J, Khadir A, Liu J, Peng H: Complement C5b-9 membrane attack complex increases expression of endoplasmic reticulum stress proteins in glomerular epithelial cells. J Biol Chem 2002; 277:41342-41351.

-18 Cybulsky AV, Takano T, Papillon J, Bijian K: Role of the endoplasmic reticulum unfolded protein response in glomerular epithelial cell injury. J Biol Chem 2005;280:2439624403.

-19 Nakajo A, Khoshnoodi J, Takenaka H, Hagiwara E, Watanabe T, Kawakami H, Kurayama R, Sekine Y, Bessho F, Takahashi S, Swiatecka-Urban A, Tryggvason K, Yan K: Mizoribine corrects defective nephrin biogenesis by restoring intracellular energy balance. J Am Soc Nephrol 2007;18:25542564.

20 Fujii Y, Khoshnoodi J, Takenaka H, Hosoyamada M, Nakajo A, Bessho F, Kudo A, Takahashi S, Arimura Y, Yamada A, Nagasawa T, Ruotsalainen V, Tryggvason K, Lee AS, Yan K: The effect of dexamethasone on defective nephrin transport caused by ER stress: a potential mechanism for the therapeutic action of glucocorticoids in the acquired glomerular diseases. Kidney Int 2006; 69:1350-1359.

-21 Inagi R, Kumagai T, Nishi H, Kawakami T, Miyata T, Fujita T, Nangaku M: Preconditioning with endoplasmic reticulum stress ameliorates mesangioproliferative glomerulonephritis. J Am Soc Nephrol 2008;19:915922.

-22 Zinszner H, Kuroda M, Wang X, Batchvarova N, Lightfoot RT, Remotti H, Stevens JL, Ron D: CHOP is implicated in programmed cell death in response to impaired function of the endoplasmic reticulum. Genes Dev 1998;12:982-995.

-23 Xu Q, Reed JC: Bax inhibitor-1, a mammalian apoptosis suppressor identified by functional screening in yeast. Mol Cell 1998;1: 337-346.

-24 Ohse T, Inagi R, Tanaka T, Ota T, Miyata T, Kojima I, Ingelfinger JR, Ogawa S, Fujita T, Nangaku M: Albumin induces endoplasmic reticulum stress and apoptosis in renal proximal tubular cells. Kidney Int 2006;70:14471455. SK: Perturbations in maturation of secretory proteins and their association with endoplasmic reticulum chaperones in a cell culture model for epithelial ischemia. Proc Nat Acad Sci USA 1996;93:8584-8589.

26 Bando Y, Tsukamoto Y, Katayama T, Ozawa K, Kitao Y, Hori O, Stern DM, Yamauchi A, Ogawa S: ORP150/HSP12A protects renal tubular epithelium from ischemia-induced cell death. FASEB J 2004;18:1401-1403.

27 Bailly-Maitre B, Fondevila C, Kaldas F, Droin N, Luciano F, Ricci JE, Croxton R, Krajewska M, Zapata JM, Kupiec-Weglinski JW, Farmer D, Reed JC: Cytoprotective gene bi-1 is required for intrinsic protection from endoplasmic reticulum stress and ischemia-reperfusion injury. Proc Natl Acad Sci USA 2006;103:2809-2814.

28 Kimura K, Jin H, Ogawa M, Aoe T: Dysfunction of the ER chaperone $\mathrm{BiP}$ accelerates the renal tubular injury. Biochem Biophys Res Commun 2008;366:1048-1053.

29 Lindenmeyer MT, Rastaldi MP, Ikehata M, Neusser MA, Kretzler M, Cohen CD, Schlöndorff D: Proteinuria and hyperglycemia induce endoplasmic reticulum stress. J Am Soc Nephrol 2008;19:2225-2236.

30 Lorz C, Justo P, Sanz A, Subira D, Egido J, Ortiz A: Paracetamol-induced renal tubular injury: a role for ER stress. J Am Soc Nephrol 2004;15:380-389.

31 Liu H, Baliga R: Endoplasmic reticulum stress-associated caspase 12 mediates cisplatin-induced LLC-PK1 cell apoptosis. J Am Soc Nephrol 2005;16:1985-1992.

32 Peyrou M, Hanna PE, Cribb AE: Cisplatin, gentamicin, and p-aminophenol induce markers of endoplasmic reticulum stress in the rat kidneys. Toxicol Sci 2007;99:346353.

33 Pallet N, Bouvier N, Legendre C, Gilleron J, Codogno P, Beaune P, Thervet E, Anglicheau D: Autophagy protects renal tubular cells against cyclosporine toxicity. Autophagy 2008;4:783-791

34 Koumenis C: ER stress, hypoxia tolerance and tumor progression. Curr Mol Med 2006 6:55-69.

35 Lee AS: GRP78 induction in cancer: therapeutic and prognostic implications. Cancer Res 2007;67:3496-3499.

36 Orrenius S, Zhivotovsky B, Nicotera P: Regulation of cell death: the calcium-apoptosis link. Nat Rev Mol Cell Biol 2003;4:552-565.

-37 Endo M, Mori M, Akira S, Gotoh T: C/EBP homologous protein (CHOP) is crucial for the induction of caspase-11 and the pathogenesis of lipopolysaccharide-induced inflammation. J Immunol 2006; 176:62456253.

38 Paschen W, Mengesdorf T: Cellular abnormalities linked to endoplasmic reticulum dysfunction in cerebrovascular disease therapeutic potential. Pharmacol Ther 2005; 108:362-375.
39 Oida Y, Izuta H, Oyagi A, Shimazawa M, Kudo T, Imaizumi K, Hara $\mathrm{H}$ : Induction of $\mathrm{BiP}$, an ER-resident protein, prevents the neuronal death induced by transient forebrain ischemia in gerbil. Brain Res 2008; 1208:217-224.

40 Glembotski CC: The role of the unfolded protein response in the heart. J Mol Cell Cardiol 2008;44:453-459.

41 Myoishi M, Hao H, Minamino T, Watanabe K, Nishihira K, Hatakeyama K, Asada Y, Okada K, Ishibashi-Ueda H, Gabbiani G, Bochaton-Piallat ML, Mochizuki N, Kitakaze M: Increased endoplasmic reticulum stress in atherosclerotic plaques associated with acute coronary syndrome. Circulation 2007; 116:1226-1233.

42 Hayashi T, Saito A, Okuno S, Ferrand-Drake M, Dodd RL, Nishi T, Maier CM, Kinouchi $\mathrm{H}$, Chan PH: Oxidative damage to the endoplasmic reticulum is implicated in ischemic neuronal death. J Cereb Blood Flow Metabol 2003;23:1117-1128.

43 Yokouchi M, Hiramatsu N, Hayakawa K, Okamura M, Du S, Kasai A, Takano Y, Shitamura A, Shimada T, Yao J, Kitamura M: Involvement of selective reactive oxygen species upstream of proapoptotic branches of unfolded protein response. J Biol Chem 2008;283:4252-4260.

44 Cullinan SB, Diehl JA: Coordination of ER and oxidative stress signaling: the PERK/ Nrf2 signaling pathway. Int J Biochem Cell Biol 2006;38:317-332.

45 De Gracia DJ, Montie HL: Cerebral ischemia and the unfolded protein response. J Neurochem 2004;91:1-8.

46 Kohno K, Higuchi T, Ohta S, Kohno K, Kumon Y, Sakaki S: Neuroprotective nitric oxide synthase inhibitor reduces intracellular calcium accumulation following transient global ischemia in the gerbil. Neurosci Lett 1997;224:17-20.

47 Zhang K, Kaufman RJ: From endoplasmicreticulum stress to the inflammatory response. Nature 2008;454:455-462.

48 Lin W, Harding HP, Ron D, Popko B: Endoplasmic reticulum stress modulates the response of myelinating oligodendrocytes to the immune cytokine interferon- $\gamma$. J Cell Biol 2005;169:603-612.

-49 Nagaraju K, Casciola-Rosen L, Lundberg I, Rawat R, Cutting S, Thapliyal R, Chang J, Dwivedi S, Mitsak M, Chen YW, Plotz P, Rosen A, Hoffman E, Raben N: Activation of the endoplasmic reticulum stress response in autoimmune myositis: potential role in muscle fiber damage and dysfunction. Arthritis Rheum 2005;52:1824-1835.

$\checkmark 50$ Kaser A, Lee AH, Franke A, Glickman JN, Zeissig S, Tilg H, Nieuwenhuis EE, Higgins DE, Schreiber S, Glimcher LH, Blumberg RS: XBP1 links ER stress to intestinal inflammation and confers genetic risk for human inflammatory bowel disease. Cell 2008;134: 743-756. 
51 Wouters BG, Koritzinsky M: Hypoxia signalling through mTOR and the unfolded protein response in cancer. Nat Rev Cancer 2008;8:851-864.

-52 Morris JA, Dorner AJ, Edwards CA, Hendershot LM, Kaufman RJ: Immunoglobulin binding protein (BiP) function is required to protect cells from endoplasmic reticulum stress but is not required for the secretion of selective proteins. J Biol Chem 1997;272: 4327-4334.

53 Peyrou M, Cribb AE: Effect of endoplasmic reticulum stress preconditioning on cytotoxicity of clinically relevant nephrotoxins in renal cell lines. Toxicol In Vitro 2007;21: 878-886.

54 Prachasilchai W, Sonoda H, Yokota-Ikeda N, Oshikawa S, Aikawa C, Uchida K, Ito K, Kudo T, Imaizumi K, Ikeda M: A protective role of unfolded protein response in mouse ischemic acute kidney injury. Eur J Pharmacol 2008;592:138-145.
55 Asmellash S, Stevens JL, Ichimura T: Modulating the endoplasmic reticulum stress response with trans-4,5-dihydroxy-1,2-dithiane prevents chemically induced renal injury in vivo. Toxicol Sci 2005;88:576-584.

56 Boyce M, Bryant KF, Jousse C, Long K, Harding HP, Scheuner D, Kaufman RJ, Ma D, Coen DM, Ron D, Yuan J: A selective inhibitor of eIF $2 \alpha$ dephosphorylation protects cells from ER stress. Science 2005;307:935939.

57 Kim I, Shu CW, Xu W, Shiau CW, Grant D, Vasile S, Cosford ND, Reed JC: Chemical biology investigation of cell death pathways activated by endoplasmic reticulum stress reveals cytoprotective modulators of ASK1. J Biol Chem 2009;284:1593-1603.

58 Qi X, Hosoi T, Okuma Y, Kaneko M, Nomura Y: Sodium 4-phenylbutyrate protects against cerebral ischemic injury. Mol Pharmacol 2004;66:899-908.

59 Liu XL, Doné SC, Yan K, Kilpeläinen P, Pikkarainen T, Tryggvason K: Defective trafficking of nephrin missense mutants rescued by a chemical chaperone. J Am Soc Nephrol 2004;15:1731-1738.
60 Xie Q, Khaoustov VI, Chung CC, Sohn J, Krishnan B, Lewis DE, Yoffe B: Effect of tauroursodeoxycholic acid on endoplasmic reticulum stress-induced caspase-12 activation. Hepatology 2002;36:592-601.

61 Ozcan U, Yilmaz E, Ozcan L, Furuhashi M, Vaillancourt E, Smith RO, Gorgun CZ, Hotamisligil GS: Chemical chaperones reduce ER stress and restore glucose homeostasis in a mouse model of type 2 diabetes. Science 2006;313:1137-1140.

62 Chen Y, Liu CP, Xu KF, Mao XD, Lu YB, Fang L, Yang JW, Liu C: Effect of taurine-conjugated ursodeoxycholic acid on endoplasmic reticulum stress and apoptosis induced by advanced glycation end products in cultured mouse podocytes. Am J Nephrol 2008;28: 1014-1022.

63 Izuhara Y, Nangaku M, Takizawa S, Takahashi S, Shao J, Oishi H, Kobayashi $H$, van Ypersele de Strihou C, Miyata T: A novel class of advanced glycation inhibitors ameliorates renal and cardiovascular damage in experimental rat models. Nephrol Dial Transplant 2008;23:497-509. 